ANNALES

POLONICI MATHEMATICI

$98.3(2010)$

\title{
On the value distribution of differential polynomials of meromorphic functions
}

\author{
by YAN XU and HuILing QIU (Nanjing)
}

\begin{abstract}
Let $f$ be a transcendental meromorphic function of infinite order on $\mathbb{C}$, let $k \in \mathbb{N}$ and $\varphi=R e^{P}$, where $R \not \equiv 0$ is a rational function and $P$ is a polynomial, and let $a_{0}, a_{1}, \ldots, a_{k-1}$ be holomorphic functions on $\mathbb{C}$. If all zeros of $f$ have multiplicity at least $k$ except possibly finitely many, and $f=0 \Leftrightarrow f^{(k)}+a_{k-1} f^{(k-1)}+\cdots+a_{0} f=0$, then $f^{(k)}+a_{k-1} f^{(k-1)}+\cdots+a_{0} f-\varphi$ has infinitely many zeros.
\end{abstract}

1. Introduction. Let $f$ and $g$ be meromorphic functions on $\mathbb{C}$, and let $a, b$ be two complex numbers. If $g=b$ whenever $f=a$, we write $f=a \Rightarrow$ $g=b$. If $f=a \Rightarrow g=b$ and $g=b \Rightarrow f=a$, we write $f=a \Leftrightarrow g=b$. The order $\rho(f)$ (see [8, 14]) of the meromorphic function $f$ is defined as

$$
\rho(f)=\varlimsup_{r \rightarrow \infty} \frac{\log T(r, f)}{\log r} .
$$

In 1959, Hayman [7] proved the following result, which is known as Hayman's Alternative.

TheOREM A. Let $f$ be a transcendental meromorphic function on $\mathbb{C}$. Then either $f$ assumes every finite value infinitely often, or every derivative of $f$ assumes every finite nonzero value infinitely often.

This result has undergone various extensions (see 1, 2, 4, 5, 9, 11, 12, 13, etc.). In 2001, Fang [5] proved the following result for functions of infinite order.

THEOREM B. Let $f$ be a transcendental meromorphic function of infinite order on $\mathbb{C}$. If $f=0 \Leftrightarrow f^{\prime}=0$, then $f^{\prime}-b(z)$ has infinitely many zeros for any $b(z) \in S$, where $S=\left\{a z^{n}: a \in \mathbb{C} \backslash\{0\}, n=0,1,2, \ldots\right\}$.

In 2005, the first author [12] proved

2010 Mathematics Subject Classification: Primary 30D35.

Key words and phrases: meromorphic function, Zalcman's lemma, infinite order, zero. 
TheOREM C. Let $f$ be a transcendental meromorphic function on $\mathbb{C}$, and let $R(\not \equiv 0)$ be a rational function and $k \in \mathbb{N}$. Suppose that all zeros of $f$ have multiplicity at least $k$ except possibly finitely many, and $f=0 \Leftrightarrow f^{(k)}=0$. Then $f^{(k)}-R$ has infinitely many zeros.

A natural problem arises: Can the rational function $R$ in Theorem $C$ be replaced by a more general meromorphic function? In this paper, for the case of $f$ with infinite order, we prove the following result.

THEOREM 1. Let $f$ be a transcendental meromorphic function of infinite order on $\mathbb{C}$, let $k \in \mathbb{N}$ and $\varphi=R e^{P}$, where $R \not \equiv 0$ is a rational function and $P$ is a polynomial, and let $a_{0}, a_{1}, \ldots, a_{k-1}$ be holomorphic functions on $\mathbb{C}$. Set

$$
L[f]:=f^{(k)}+a_{k-1} f^{(k-1)}+\cdots+a_{0} f .
$$

Suppose that all zeros of $f$ have multiplicity at least $k$ except possibly finitely many, and $f=0 \Leftrightarrow L[f]=0$. Then $L[f]-\varphi$ has infinitely many zeros.

REMARK 1. Obviously, the assumption "all zeros of $f$ have multiplicity at least $k$, and $f=0 \Leftrightarrow L[f]=0$ " is equivalent to "all zeros of $f$ have multiplicity at least $k+1$, and $f=0 \Leftarrow L[f]=0$ ".

THEOREM 2. Let $f$ be a transcendental meromorphic function of infinite order on $\mathbb{C}$, let $k \in \mathbb{N}$ and $\varphi=R e^{P}$, where $R \not \equiv 0$ is a rational function and $P$ is a polynomial, and let $a_{0}, a_{1}, \ldots, a_{k-1}$ be holomorphic functions on $\mathbb{C}$. If $f$ has only finitely many zeros, then $L[f]-\varphi$ has infinitely many zeros, where $L[f]$ is defined in $(*)$.

From Theorems 1 and 2, we get

COROLlaRY 1. Let $f$ be a transcendental meromorphic function of infinite order on $\mathbb{C}$, let $k \in \mathbb{N}$ and $\varphi=R e^{P}$, where $R \not \equiv 0$ is a rational function and $P$ is a polynomial. Suppose that all zeros of $f$ have multiplicity at least $k$ except possibly finitely many, and $f=0 \Leftrightarrow f^{(k)}=0$. Then $f^{(k)}-\varphi$ has infinitely many zeros.

COROLlaRY 2. Let $f$ be a transcendental meromorphic function of infinite order on $\mathbb{C}$, let $k \in \mathbb{N}$ and $\varphi=R e^{P}$, where $R \not \equiv 0$ is a rational function and $P$ is a polynomial. If $f$ has only finitely many zeros, then $f^{(k)}-\varphi$ has infinitely many zeros.

REMARK 2. As Hayman's inequality [7, 8] for small functions is still unknown, Theorem 2 and Corollary 2 are not direct consequences of Hayman's inequality.

2. Some lemmas. The following three lemmas are due to Liu, Nevo and Pang [9]. 
LEMma 1. Let $k$ be a positive integer and let $\left\{f_{n}\right\}$ be a family of functions meromorphic on $\Delta=\{z:|z|<1\}$, all of whose zeros have multiplicity at least $k+1$. If $a_{n} \rightarrow a,|a|<1$, and $f_{n}^{\#}\left(a_{n}\right) \rightarrow \infty$, then there exist a subsequence of $\left\{f_{n}\right\}$ (which we still write as $\left\{f_{n}\right\}$ ), a sequence of points $z_{n} \in D, z_{n} \rightarrow z_{0},\left|z_{0}\right|<1$, and a sequence of positive numbers $\rho_{n} \rightarrow 0$ such that

$$
g_{n}(\zeta)=\frac{f_{n}\left(z_{n}+\rho_{n} \zeta\right)}{\rho_{n}^{k}} \rightarrow g(\zeta)
$$

locally uniformly with respect to the spherical metric, where $g$ is a nonconstant meromorphic function on $\mathbb{C}$, such that $g^{\#}(\zeta) \leq g^{\#}(0)=k+1$, and $\rho_{n} \leq M / \sqrt[k+1]{f_{n}^{\#}\left(a_{n}\right)}$, where $M$ is independent of $n$.

Here, as usual, $g^{\#}(\zeta)=\left|g^{\prime}(\zeta)\right| /\left(1+|g(\zeta)|^{2}\right)$ is the spherical derivative of $g$. The above lemma is in fact another version of Zalcman's Lemma (see [3, 10, 11, 15, 16, etc.). The main difference here is the estimate of $\rho_{n}$ in the vicinity of some point of nonnormality. Moreover, by using the AhlforsShimizu characteristic function, we can deduce (as in [10] or [11]) that the limit function $g$ in Lemma 1 has order at most 2 since $g^{\#}(\zeta) \leq g^{\#}(0)=k+1$.

Lemma 2. Let $f$ be a meromorphic function of infinite order on $\mathbb{C}$. Then there exist points $z_{n} \rightarrow \infty$ such that for every $N>0, f^{\#}\left(z_{n}\right)>\left|z_{n}\right|^{N}$ if $n$ is sufficiently large.

Lemma 3. Let $R(z) \not \equiv 0$ be a rational function. Then there exists $k>0$ such that $\left|z R^{\prime}(z)\right| \leq k|R(z)|$ for large enough $z$.

The next lemma is due to Fang [5] and Fang-Zalcman [6].

Lemma 4. Let $f$ be a meromorphic function of finite order on $\mathbb{C}, b a$ nonzero complex number, and $k$ a positive integer. If all zeros of $f$ have multiplicity at least $k, f=0 \Leftrightarrow f^{(k)}=0$, and $f^{(k)} \neq b$, then $f$ is a constant.

\section{Proofs of theorems}

Proof of Theorem 1. Suppose that $L[f](z)-\varphi(z)$ has finitely many zeros. Then, for large $z$, we have

$$
\frac{L[f](z)}{\varphi(z)} \neq 1
$$

Set

$$
F(z)=f(z) / \varphi(z) .
$$

Obviously, the order of $F$ is equal to that of $f$, and so $F$ is of infinite order. By Lemma 2, there exist points $z_{n} \rightarrow \infty$ such that for every $N>0$ and sufficiently large $n$ we have $F^{\#}\left(z_{n}\right)>\left|z_{n}\right|^{N}$. Noting that $\varphi(z)$ has only 
finitely many zeros and poles, we find that all zeros of $F\left(z+z_{n}\right)$ (for large $n$ ) in $\Delta$ have multiplicity at least $k+1$.

Then, by Lemma 1, there exist a subsequence of $\left\{F\left(z+z_{n}\right)\right\}$ (without loss of generality, we may still write it as $F\left(z+z_{n}\right)$ ), a sequence of points $z_{n}^{\prime} \rightarrow z_{0}$ and $\left|z_{0}\right|<1$, and a sequence of positive numbers $\rho_{n} \rightarrow 0$ such that $\rho_{n} \leq M / \sqrt[k+1]{F^{\#}\left(z_{n}\right)}$ and

$$
g_{n}(\zeta)=\frac{F\left(z_{n}+z_{n}^{\prime}+\rho_{n} \zeta\right)}{\rho_{n}^{k}} \rightarrow g(\zeta)
$$

locally uniformly with respect to the spherical metric, where $g$ is a nonconstant meromorphic function on $\mathbb{C}$, and $M$ is independent of $n$. Moreover, $g$ is of order at most 2. By Hurwitz's theorem, all zeros of $g$ have multiplicity at least $k+1$.

By simple calculation, for $0 \leq i \leq k$, we have

$$
F^{(i)}(z)=\frac{f^{(i)}(z)}{\varphi(z)}-\sum_{j=1}^{i}\left(\begin{array}{l}
i \\
j
\end{array}\right) F^{(i-j)}(z) \frac{\varphi^{(j)}(z)}{\varphi(z)} .
$$

Obviously, $\varphi^{(j)}(z)=\sum_{m=0}^{j}\left(\begin{array}{c}j \\ m\end{array}\right) R^{(m)}(z)\left(e^{P(z)}\right)^{(j-m)}$, so that $\varphi^{(j)}(z) / \varphi(z)$ is a polynomial of $R^{(m)}(z) / R(z)$ and $P^{(m)}(z)(m=1, \ldots, j)$. Now we rewrite (4) as

$$
F^{(i)}(z)=\frac{f^{(i)}(z)}{\varphi(z)}-\sum_{j=1}^{i} Q_{j}(z) F^{(i-j)}(z),
$$

where $Q_{j}(z)$ is a polynomial of $R^{(m)}(z) / R(z)$ and $P^{(m)}(z)(m=1, \ldots, j)$ for $j=1, \ldots, i$.

Thus, from (3) and (5), we have

$$
\begin{aligned}
\rho_{n}^{k-i} & g_{n}^{(i)}(\zeta)=F^{(i)}\left(z_{n}+z_{n}^{\prime}+\rho_{n} \zeta\right) \\
& =\frac{f^{(i)}\left(z_{n}+z_{n}^{\prime}+\rho_{n} \zeta\right)}{\varphi\left(z_{n}+z_{n}^{\prime}+\rho_{n} \zeta\right)}-\sum_{j=1}^{i} Q_{j}\left(z_{n}+z_{n}^{\prime}+\rho_{n} \zeta\right) F^{(i-j)}\left(z_{n}+z_{n}^{\prime}+\rho_{n} \zeta\right) \\
& =\frac{f^{(i)}\left(z_{n}+z_{n}^{\prime}+\rho_{n} \zeta\right)}{\varphi\left(z_{n}+z_{n}^{\prime}+\rho_{n} \zeta\right)}-\sum_{j=1}^{i} \rho_{n}^{j} Q_{j}\left(z_{n}+z_{n}^{\prime}+\rho_{n} \zeta\right) \frac{F^{(i-j)}\left(z_{n}+z_{n}^{\prime}+\rho_{n} \zeta\right)}{\rho_{n}^{j}}
\end{aligned}
$$

for $i=0,1, \ldots, k$.

Now we show that on each compact subset of $\mathbb{C}$,

$$
\lim _{n \rightarrow \infty} \rho_{n}^{j} Q_{j}\left(z_{n}+z_{n}^{\prime}+\rho_{n} \zeta\right)=0 \quad \text { for } 1 \leq j \leq i \leq k .
$$

First, by Lemma 3, we get

$$
\lim _{n \rightarrow \infty} \frac{R^{(m)}\left(z_{n}+z_{n}^{\prime}+\rho_{n} \zeta\right)}{R\left(z_{n}+z_{n}^{\prime}+\rho_{n} \zeta\right)}=0 \quad(1 \leq m \leq j) .
$$


On the other hand, for large $n$, we have

$$
P^{(m)}\left(z_{n}+z_{n}^{\prime}+\rho_{n} \zeta\right)=O\left(z_{n}^{p}\right),
$$

where $p=\max \{\operatorname{deg} P-m, 0\}$ and $1 \leq m \leq j$. Noting that, for sufficiently large $n$ and every $N>0$,

$$
\rho_{n} \leq \frac{M}{\sqrt[k+1]{F^{\#}\left(z_{n}\right)}}<M\left|z_{n}\right|^{-N /(k+1)}
$$

for any given $\alpha>0$ we have

$$
\rho_{n}^{\alpha}\left|z_{n}\right|^{p}<M^{\alpha}\left|z_{n}\right|^{p-\alpha N /(k+1)} \rightarrow 0,
$$

since we can choose $N$ so large that $p-\alpha N /(k+1)<0$. This and (8) imply that, for any given $\alpha>0$,

$$
\lim _{n \rightarrow \infty} \rho_{n}^{\alpha} P^{(m)}\left(z_{n}+z_{n}^{\prime}+\rho_{n} \zeta\right)=0 \quad \text { for } 1 \leq m \leq j,
$$

Recalling that $Q_{j}(z)$ is a polynomial of $R^{(m)}(z) / R(z)$ and $P^{(m)}(z)(m=$ $1, \ldots, j)$, from (7) and (9) we obtain (6).

We note that $F^{(i-j)}\left(z_{n}+z_{n}^{\prime}+\rho_{n} \zeta\right) / \rho_{n}^{j}$ is locally bounded on $\mathbb{C}$ minus the set of poles of $g(\zeta)$ since $F\left(z_{n}+z_{n}^{\prime}+\rho_{n} \zeta\right) / \rho_{n}^{k} \rightarrow g(\zeta)$. Then, on every compact subset of $\mathbb{C}$ which contains no poles of $g(\zeta)$, we have

$$
\frac{f^{(k)}\left(z_{n}+z_{n}^{\prime}+\rho_{n} \zeta\right)}{\varphi\left(z_{n}+z_{n}^{\prime}+\rho_{n} \zeta\right)} \rightarrow g^{(k)}(\zeta)
$$

and

$$
\frac{f^{(i)}\left(z_{n}+z_{n}^{\prime}+\rho_{n} \zeta\right)}{\varphi\left(z_{n}+z_{n}^{\prime}+\rho_{n} \zeta\right)} \rightarrow 0
$$

for $i=0,1, \ldots, k-1$, and thus

$$
\frac{L[f]\left(z_{n}+z_{n}^{\prime}+\rho_{n} \zeta\right)}{\varphi\left(z_{n}+z_{n}^{\prime}+\rho_{n} \zeta\right)} \rightarrow g^{(k)}(\zeta),
$$

since $a_{0}, \ldots, a_{k-1}$ are holomorphic.

We claim

(i) $g(\zeta)=0 \Leftrightarrow g^{(k)}(\zeta)=0$;

(ii) $g^{(k)} \neq 1$ on $\mathbb{C}$.

Obviously, $g(\zeta)=0 \Rightarrow g^{(k)}(\zeta)=0$. Now suppose $g^{(k)}\left(\zeta_{0}\right)=0$. Since all zeros of $g(\zeta)$ have multiplicity at least $k+1$, we know that $g^{(k)}(\zeta) \not \equiv 0$. Hurwitz's theorem implies that there exist $\zeta_{n} \rightarrow \zeta_{0}$ such that (for $n$ sufficiently large)

$$
L[f]\left(z_{n}+z_{n}^{\prime}+\rho_{n} \zeta_{n}\right)=0 .
$$

It follows that $f\left(z_{n}+z_{n}^{\prime}+\rho_{n} \zeta_{n}\right)=0$. Hence $g\left(\zeta_{0}\right)=\lim _{n \rightarrow \infty} g_{n}\left(\zeta_{n}\right)=0$. So $g^{(k)}(\zeta)=0 \Rightarrow g(\zeta)=0$. This proves (i). 
Next we prove (ii). From (1) and (10), Hurwitz's theorem shows that on $\mathbb{C}$ minus the poles of $g$, the derivative $g^{(k)}$ is either identically 1 , or never equal to 1 . Clearly, the same alternative also holds on the whole $\mathbb{C}$. If $g^{(k)}(\zeta) \equiv 1$, then $g$ is a polynomial of degree $k$. But this contradicts the fact all zeros of $g$ have multiplicity at least $k+1$. So we get (ii).

Thus by Lemma 4, $g$ must be a constant, contradiction. This completes the proof of Theorem 1 .

Proof of Theorem 2. Since $f$ has only finitely many zeros, by applying Hurwitz's theorem, we deduce from (3) that $g \neq 0$. Then, by using the same argument as in the proof of Theorem 1, we can prove Theorem 2. Here we omit the details.

Acknowledgments. We wish to thank the referee for his/her valuable comments and suggestions.

The first author was supported by NSFC (Grant No. 10871094).

\section{References}

[1] W. Bergweiler, On the product of a meromorphic function and its derivatives, Bull. Hong Kong Math. Soc. 1 (1997), 97-101.

[2] W. Bergweiler and X. C. Pang, On the derivatives of meromorphic functions with multiple zeros, J. Math. Anal. Appl. 278 (2003), 285-292.

[3] H. H. Chen and Y. X. Gu, An improvement of Marty's criterion and its applications, Sci. China Ser. A (6) 36 (1993), 674-681.

[4] M. L. Fang, A note on a problem of Hayman, Analysis 20 (2000), 45-49.

[5] -, Picard values and normality criterion, Bull. Korean Math. Soc. (2) 38 (2001), 379-387.

[6] M. L. Fang and L. Zalcman, Normal families and shared values of meromorphic functions, Ann. Polon. Math. 80 (2003), 133-141.

[7] W. K. Hayman, Picard values of meromorphic functions and their derivatives, Ann. of Math. 70 (1959), 9-42.

[8] -, Meromorphic Functions, Clarendon Press, Oxford, 1964.

[9] X. J. Liu, S. Nevo and X. C. Pang, On the kth derivative of meromorphic functions with zeros of multiplicity at least $k+1$, J. Math. Anal. Appl. 348 (2008), 516-529.

[10] X. C. Pang and L. Zalcman, Normal families and shared values, Bull. London Math. Soc. 32 (2000), 325-331.

[11] Y. F. Wang and M. L. Fang, Picard values and normal families of meromorphic functions with multiple zeros, Acta Math. Sinica (N.S.) 14 (1998), 17-26.

[12] Y. Xu, On the value distribution of derivatives of meromorphic functions, Appl. Math. Lett. 18 (2005), 597-602.

[13] - , Picard values and derivatives of meromorphic functions, Kodai Math. J. 28 (2005), 99-105.

[14] L. Yang, Value Distribution Theory, Springer \& Sci. Press, Berlin, 1993.

[15] L. Zalcman, A heuristic principle in complex function theory, Amer. Math. Monthly 82 (1975), 813-817. 
[16] L. Zalcman, Normal families: new perspectives, Bull. Amer. Math. Soc. 35 (1998), 215-230.

Yan Xu

Department of Mathematics Nanjing Normal University

Nanjing 210046, P.R. China

E-mail: xuyan@njnu.edu.cn
Huiling Qiu Department of Applied Mathematics Nanjing Audit University Nanjing 210029, P.R. China E-mail: qiuhuiling1304@sina.com

Received 17.9.2009

and in final form 12.11.2009 
